\title{
Spatial Selection and Local Adaptation Jointly Shape Life-History Evolution during Range Expansion
}

\author{
Katrien H. P. Van Petegem, ${ }^{1, *, \dagger}$ Jeroen Boeye, ${ }^{1, \dagger}$ Robby Stoks, ${ }^{2}$ and Dries Bonte ${ }^{1}$ \\ 1. Department of Biology, Terrestrial Ecology Unit, Ghent University, K. L. Ledeganckstraat 35, B-9000 Ghent, Belgium; 2. Laboratory of \\ Aquatic Ecology, Evolution, and Conservation, KU Leuven, Charles Deberiotstraat 32, B-3000 Leuven, Belgium \\ Submitted October 6, 2015; Accepted July 5, 2016; Electronically published September 19, 2016 \\ Online enhancements: appendix, supplemental files. Dryad data: http://dx.doi.org/10.5061/dryad.n0c67.
}

\begin{abstract}
In the context of climate change and species invasions, range shifts increasingly gain attention because the rates at which they occur in the Anthropocene induce rapid changes in biological assemblages. During range shifts, species experience multiple selection pressures. For poleward expansions in particular, it is difficult to interpret observed evolutionary dynamics because of the joint action of evolutionary processes related to spatial selection and to adaptation toward local climatic conditions. To disentangle the effects of these two processes, we integrated stochastic modeling and data from a common garden experiment, using the spider mite Tetranychus urticae as a model species. By linking the empirical data with those derived form a highly parameterized individual-based model, we infer that both spatial selection and local adaptation contributed to the observed latitudinal lifehistory divergence. Spatial selection best described variation in dispersal behavior, while variation in development was best explained by adaptation to the local climate. Divergence in life-history traits in species shifting poleward could consequently be jointly determined by contemporary evolutionary dynamics resulting from adaptation to the environmental gradient and from spatial selection. The integration of modeling with common garden experiments provides a powerful tool to study the contribution of these evolutionary processes on life-history evolution during range expansion.
\end{abstract}

Keywords: global change, Tetranychus urticae, quantitative genetic trait divergence, pattern-oriented modeling, dispersal evolution, sawtooth pattern.

\section{Introduction}

Numerous species are currently shifting their ranges because of contemporary climate change (Parmesan 2006) or are expanding them after being introduced in a new environment (Richardson and Rejmanek 2011). During such

\footnotetext{
* Corresponding author; e-mail: katrien.vanpetegem@ugent.be.

$\dagger$ These authors contributed equally to this work. ORCIDs: Bonte, 0000-0002-3320-7505.
}

Am. Nat. 2016. Vol. 188, pp. 485-498. (C) 2016 by The University of Chicago. 0003-0147/2016/18805-56566\$15.00. All rights reserved.

DOI: $10.1086 / 688666$ shifts and range expansions, species undergo multiple selection pressures (Phillips et al. 2010). Especially for poleward range shifts or expansions, a straightforward interpretation of the observed evolutionary dynamics is hampered because of the simultaneous evolutionary responses to the changing local environmental conditions and to the expansion process per se.

Species expanding or shifting their range poleward experience a change in temperature and growing season that could affect their life histories. The lower temperatures in northern regions can affect species' diapause behavior (e.g., the Colorado potato beetle; see Piiroinen et al. 2011; Lehmann et al. 2014, 2015), and especially in multivoltine species, changes in the length of the growing season have an impact on development time, growth rate, and adult size. Because of the gradual shortening of the growing season, a gradually faster development is needed to attain an equal number of generations within this decreasing time frame. At a certain point, however, development speed is at is maximum. At this point, voltinism abruptly decreases (i.e., loss of a generation), in turn allowing a sudden relaxation of development speed. This mechanism of a step-by-step reduction in voltinism with increasing latitude creates a typical sawtooth pattern in development time (i.e., alterations of gradual increase and sudden decrease in development speed; see Roff 1980; Kivela et al. 2011; Levy et al. 2015). Furthermore, high-latitude populations tend to compensate for the low temperatures that plastically reduce growth rate through the evolution of genetically faster growth rates (i.e., countergradient variation; see Conover and Schultz 1995). In some instances, development time can moreover share an underlying mechanism with growth rate (Kivela et al. 2011), and together these two traits can impact adult size, leading to either bigger, smaller, or equal-sized individuals in more northern regions (Blanckenhorn and Demont 2004). Apart from these climatic changes, many range-shifting species may also suffer from changes in habitat quality and quantity. However, this is mainly restricted to native range climate-tracking species (as 
opposed to invasive species), for which deteriorating habitat is one of the main explanations for the occurrence of their initial range limits (North et al. 2011; Hargreaves et al. 2014).

On top of this pressure to adjust to the changing local environment, the process of range expansion in itself entails a strong selection pressure. First, since the most dispersive phenotypes accumulate at the expansion front, assortative mating takes place (Phillips et al. 2010; Shine et al. 2011). This results in increased dispersal abilities at the range front, as has been illustrated theoretically (e.g., Travis and Dytham 2002; Burton et al. 2010; Perkins et al. 2013) as well as empirically through field and common garden studies (e.g., Phillips et al. 2006; Mitikka and Hanski 2010; Hill et al. 2011; Huang et al. 2015) and experimental evolution (Fronhofer and Altermatt 2015). Dispersal evolution thus affects (Kubisch et al. 2014) and is affected by range expansion (reviewed in Hill et al. 2011). Second, because of the locally low densities at the leading edge, individuals in the vanguard of an expanding range are predicted to experience $r$ rather than $K$ selection, which would translate into a higher investment in reproduction (Phillips 2009; Phillips et al. 2010). Range expansion thus results in a positive selection for dispersal because of the interaction between spatial sorting (and thus assortative mating) of dispersive phenotypes and an increased population growth rate driven by density release at the expansion front. Through both mechanisms, range expansion therefore contributes to a process of spatial selection (Shine et al. 2011; Perkins et al. 2013).

Local adaptation to changing climatic conditions and spatial selection can thus jointly impact evolutionary dynamics in species expanding poleward. However, we lack a clear understanding of their relative importance in shaping quantitative genetic trait differentiation along latitudinal gradients. For example, a greater investment in thorax mass in northern populations of an insect species can result from spatial selection (dispersiveness is selected for at the range front) as well as from local adaptation (lower temperatures might decrease muscle efficiency). Likewise, increased dispersal in plant populations near the range edge could purely result from mechanisms to avoid inbreeding (adaptation to low mate availability; Hargreaves and Eckert 2014) but could also be caused by spatial selection. Insights are, to date, mainly derived from theory (Perkins et al. 2013; Hargreaves et al. 2015) or from correlative - often phenotypic - approaches (Therry et al. 2014a, $2014 b, 2014 c, 2015)$.

Here, we combine common garden breeding and an individual-based model (IBM) to study the putative causes of multivariate trait evolution during poleward range expansion. With a full life-history perspective, we assess latitudinal quantitative genetic trait differentiation in the two-spotted spider mite Tetranychus urticae Koch (Acari, Tetranychi- dae), which has recently expanded its European range. By contrasting empirical patterns in life-history trait divergence with those derived from a stochastic IBM, we are able to determine whether this trait divergence is best explained by only local adaptation, only spatial selection, or their joint action.

\section{Material and Methods}

\section{Life-History Evolution along the Sampled Gradient}

Study Species. The herbivorous spider mite Tetranychus urticae is an agricultural pest species with a worldwide distribution. It reproduces through arrhenotokous parthenogenesis, whereby unfertilized eggs develop into males and fertilized eggs into females. Sex ratio in T. urticae is usually female biased (3:1; Krainacker and Carey 1989), but mothers can alter the sex ratio of their young (Young et al. 1986). Each female may produce more than 50 female offspring, and at optimal temperatures $\left(27^{\circ}-30^{\circ} \mathrm{C}\right)$, mites can complete their life cycle in 8-10 days (Sabelis 1981). The species can engage in aerial long-distance dispersal (making use of aerial currents), which is preceded by a unique predispersal behavior. Like many arthropods, T. urticae can go into diapause when conditions are suboptimal (e.g., food shortage, desiccation, cold). This ability is restricted to the adult stage of the species. From approximately 1983 onward, the mite species has expanded its European range from the Mediterranean to (at least) southern Scandinavia (K. H. P. Van Petegem, personal observation); this occurred at least partially through aerial long-distance dispersal (a detailed description of the mite's range expansion can be found in Carbonnelle et al. 2007).

Population Sampling. We collected spider mites during the summers of 2011 and 2012 along an $800-\mathrm{km}$ latitudinal gradient from northwestern Belgium $\left(51.1^{\circ} \mathrm{N}\right)$ to northern Denmark $\left(57.7^{\circ} \mathrm{N}\right.$; fig. 1$)$. To minimize variation due to adaptation to different host plant species and human pressure (e.g., harvesting, pesticides) and to maximize latitudinal, climatic variation relative to variation in continentality (i.e., longitudinal variation), we searched for mites on a small selection of host plants within (semi-)natural area along the coast (see "Field Collection Sites," available online). In 2011, we collected spider mites in 20 sites. In 2012, we collected them in 12 out of these 20 sites, thereby omitting populations that were very close to one another. To avoid mites being in common garden conditions too long (allowing domestication), trait assessments were split up over two consecutive years. Diapause incidence, longevity, fecundity, egg survival, juvenile survival, and development time were assessed with mites collected in 2011, while dispersal propensity, dispersal latency, sex ratio, and adult size were assessed with mites collected in 2012. 


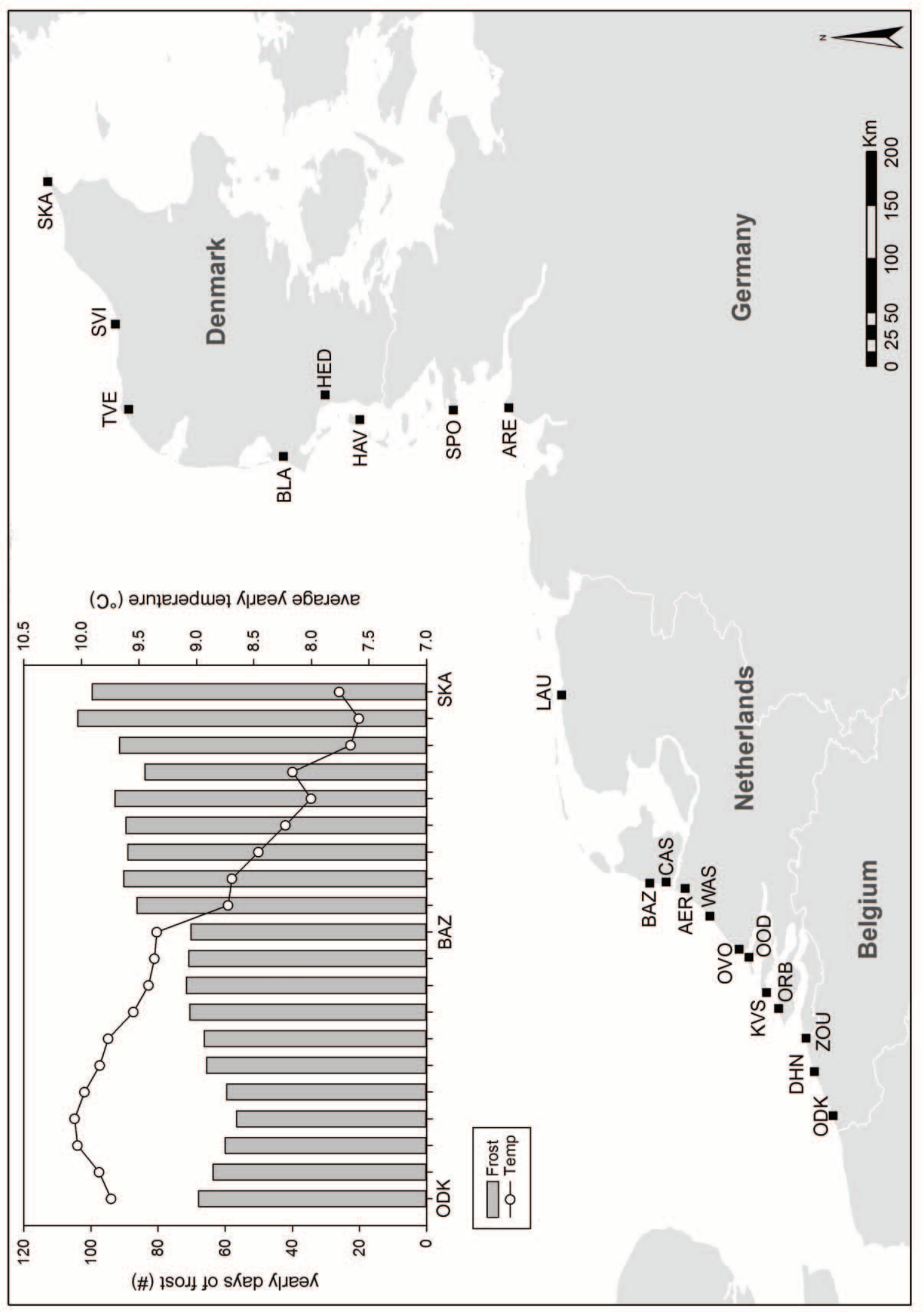

현훙.

艺

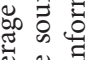

एँ

ฐ

들

뭉

密

总

पे छี

离䒕

콩 믕

芯芯

䨌

छ సे

है प्ष

त

듈

敬寻

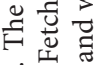

艺 㝘

纯

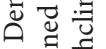

可 完过

文行

苟芒

可

कै.

현

ث

乙

声蓄

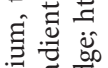

के

๑ 콣

\&

需节

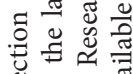

융

पु 을 원

氙芯芯

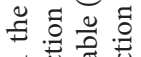

न

웅 $>0$

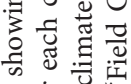

원휴.

昰高

$\because$ 矛过

จ 
Common Garden and Synchronization. Common garden breeding techniques are robust methods for detecting genetic variation among populations. In both 2011 and 2012, a common garden stock population was thus generated in the lab for each collection site by putting between 50 and several hundred mites from the collection site on whole bean plants (Phaseolus vulgaris, variety Prélude). Beans are known to be a highly favorable host for T. urticae. Mites from different collection sites are therefore not expected to show substantial genetic variation in fitness on this plant species (Agrawal et al. 2002; Gotoh et al. 2004). However, though we selected our common garden conditions to be as neutral as possible, different populations (genotypes) may still have reacted differently toward these standardized conditions (see sec. A1.1; sec. A1-A6 available online). We, however, minimized this bias in our analyses by following a pattern-oriented approach, thereby avoiding a direct comparison of empirical trait values with modeled ones. The common garden stock populations were maintained at room temperature with a light regime of 16L:8D. Mites remained in these stock populations for one to four generations (with the exception of the assessment of sex ratio, where they were in common garden for about 20 generations). Subsequently, before the start of an experiment, a synchronization of the mites was performed to obtain a large pool of same-aged, mated adult females (see sec. A1.2). With the exception of diapause incidence (which was performed almost immediately after population sampling), all trait assessments were thus performed with at least thirdgeneration mites (at least one generation in the stock population, plus one generation of synchronization). The relatively short stay in common garden was chosen as a balance between excluding direct environmental effects (phenotypic plasticity, environmentally induced maternal effects) and keeping as much of the original genotypic differentiation as possible (i.e., preventing loss through adaptation to the new host [bean] or the lab environment).

Data Collection and Statistics. A detailed overview of the applied methodology during data collection can be found in sections A1.3-A1.8. In short, we measured the following 10 life-history traits for all populations: dispersal propensity, dispersal latency, diapause incidence, fecundity, longevity, adult size, egg survival, juvenile survival, sex-specific development time, and sex ratio (all resulting data are deposited in the Dryad Digital Repository: http://dx.doi.org/10.5061 /dryad.n0c67 [Van Petegem et al. 2016]). Because it was not possible to assess all traits simultaneously for one individual mite, several different, independent experimental setups were constructed. Thus, though the unit of observation was always an individual mite, the different traits were not assessed for one and the same individual. The first six traits were assessed for only female mites (not relevant for males). Using estab- lished behavioral assays (see Li and Margolies 1994; Van Petegem et al. 2015), dispersal was assessed by quantifying a female's propensity and latency (i.e., the period of decisionmaking) to perform a unique predispersal behavior tightly linked to aerial dispersal. Because dispersal is density dependent (Harrison 1980; Denno and Peterson 1995), we measured these dispersal behaviors at different densities. We furthermore obtained a population-level measure for intrinsic growth rate by multiplying values of lifetime fecundity with juvenile survival, egg survival, and $1 /$ sex ratio (i.e., ratio of daughters to total number of offspring). These values were resampled (using the sample size of sex ratio, which was the lowest of all four traits) from the quantified distributions of the respective traits.

We performed a multivariate distance-based ANOVA to test for variation in multivariate life-history parameter space (all 10 measured life-history traits), using the vegan and permute packages of RStudio (ver. R 3.2.3; RStudio Team 2015; for more detailed information, see sec. A1.9). The multivariate test showed significant variation in life-history strategies among the different populations $\left(F_{1,8}=2.23, P=.044, R^{2}=0.22\right.$ for the subset of 12 populations sampled in both 2011 and 2012 [and hence all 10 measured traits]; $F_{1,16}=3.31, P=.012, R^{2}=0.17$ for 18 of the 20 populations sampled in 2011 [and hence the subset of six traits measured for only 2011]).

This validated the use of subsequent univariate analyses, which were performed using (generalized) linear mixed models (SAS ver. 9.4; SAS Institute 2013). For each trait separately, differentiation along the latitudinal gradient was assessed. Latitude, mite density (for dispersal propensity), and host plant species were the independent variables. The patterns found for latitude were maintained when we used the local average yearly temperature or the coastal distance to the uppermost population as the independent variable instead of latitude (K. H. P. Van Petegem, unpublished data). We tested trait differentiation related to host plant identity because the dominant host plant species in the field changed with latitude and could thus have affected the latitudinal signal. In all cases, we corrected for nonindependence of our data within single populations by adding maternal line and other experimental sources of dependency as random effects (for a detailed outline of the different models, see sec. A1.9). Effective denominator degrees of freedom for the tests of fixed effects were computed according to a general Satterthwaite approximation. Because the variance explained by random effects varied among the different dependent variables in our study, these effective denominator degrees of freedom were different for each statistical model.

Additionally, because we theoretically expect a sawtooth pattern for development time, we used segmented linear regression on the population averages (Muggeo 2003, 2008), 
following multiple breakpoint analyses for data fitting. Davies's tests indicated the presence of three breakpoints. Analysis of deviance with the linear model was used to verify a putative better fit of the segmented linear regression (sawtooth) versus a linear regression (Crawley 2007). These analyses were done in RStudio (ver. R 3.2.3; RStudio Team 2015), using the segmented package (Muggeo 2008).

\section{Inferring Mechanisms by Contrasting the Empirical Data with a Parameterized Individual-Based Model}

The Individual-Based Model (IBM). We here only outline the basic principles of the IBM but provide a detailed description and motivation in section A2 (the modeling code is available in GitHub: https://github.com/jeroenboeye/Van -Petegem-et-al._Evolution-during-range-expansion_code). We designed a stochastic, individual-based, and spatially explicit model to simulate the evolutionary dynamics in T. urticae along a climatic gradient comparable to the one we studied empirically. The IBM is inspired by an IBM of Bancroft and Margolies (1999) that simulated the dynamics among T. urticae, its host plant, and its predator. We adjusted this existing IBM to simulate population dynamics at a time step basis of 1 day (for which empirical data were available) in a simplified model landscape. This landscape consisted of a grid of 100 rows (latitude) and five columns (longitude). Local population dynamics were simulated within each of these grid cells. The grid reflects a latitudinal range of 1,000 km $\left(10 \times 10-\mathrm{km}^{2}\right.$ grid cells $)$ and corresponds with the macrogeographic scale at which we sampled T. urticae in the field. The length of the growing season, determined by seasonal variation in temperature, was defined at the grid level by two trigonometric functions, which were based on actual data.

The IBM simulated the behavior and life history of adult female mites, because females are the reproducing sex and adult females the main dispersers. No mating limitations and recombinations were consequently implemented. Using data from Sabelis (1981), development, longevity, mortality, and fecundity of the mites were all simulated according to the local grid cell temperature. Mites followed a pattern of exponential growth. However, as a compromise to maintain high computational efficiency, individuals were randomly deleted as soon as more than 200 mites occupied a grid cell. We assumed density-independent aerial dispersal among grid cells, because the large spatial scale used $\left(10 \times 10-\mathrm{km}^{2}\right.$ grid cells) did not allow us to sensibly incorporate density dependence (which is important at the level of a single leaf or plant). Dispersal mortality was set relatively high and reflects transience and settlement costs (90\%; see De Roissart et al. 2015). The probability for an individual mite to engage in aerial long-distance dispersal was modeled as an unconditional nearest-neighbor dispersal rate, determined by a single locus subject to selection/muta- tion. Other traits subject to selection and mutation were development, fecundity, and the timing of diapause onset and termination. A linear trade-off between development and fecundity was implemented in order to constrain the evolutionary trajectories. This trade-off function was coded by a single-locus trait that altered the balance of investment between development and fecundity. Because no empirical data on such a trade-off are available, we tested several functions where the maximal increase or decrease in performance of either trait was limited to $10 \%$ or $20 \%$ (assumed realistic, conservative percentages). In total, four trade-off balances $(10 \%-10 \%, 10 \%-20 \%, 20 \%-10 \%, 20 \%-20 \%)$ were thus modeled. For instance, $10 \%-20 \%$ implies that a maximum $10 \%$ increase/decrease in development corresponds with a maximum $20 \%$ decrease/increase in fecundity (see sec. A2.6).

Because we aimed to contrast evolutionary dynamics resulting from spatial selection and local adaptation, we tested three competing major model scenarios: (1) a scenario of range expansion along a homogeneous gradient, (2) a scenario with range expansion along a latitudinal climatic gradient, and (3) a scenario where evolution could occur within this same heterogeneous gradient but without the process of range expansion. In the stable range scenario (scenario 3), individuals were initialized along the entire climatic gradient. For scenarios with range expansion (scenarios 1 and 2), only the 10 southernmost rows were initialized with genetically diverse individuals, thereby allowing range expansion toward the northern grid cells. This range expansion was constrained in scenario 2 by the seasonal conditions that affected development, survival, and fecundity. All simulations were run via high-performance computing (Ghent University). A total of 100 replicates were run for each of 12 specific model scenarios (three major model scenarios $x$ four trade-off balances). The IBM was halted after 100,000 time steps (i.e., when the entire range was more or less fully occupied in all eight specific model scenarios that included range expansion).

Comparison of Empirical and Simulation Results. We tested how closely the empirically observed latitudinal patterns of life-history traits matched those predicted by the IBM. More specifically, we performed goodness-of-fit tests on summary statistics of those three life-history traits that were subject to selection and for which a comparison between empirical and simulation data could be made: the regression slopes against latitude of intrinsic growth rate, dispersal, and development time, and the amplitude and wavelength of the sawteeth for development time (for more information on these five summary statistics, see sec. A3). We compared the empirical and simulation data with a pattern-oriented approach (Grimm et al. 2005), using approximate Bayesian computation to select the specific model scenarios for which 
a derived summary statistic best matched that from the empirical data (Csillery et al. 2010; Baiser et al. 2013; Wiegand and Moloney 2014). We ran three competing major model scenarios (scenarios 1-3), each of which was coupled with four implemented trade-off balances between development and fecundity (all 10\%/20\% combinations), giving a total of 12 specific model scenarios. Posterior distributions of the five summary statistics were obtained from 100 independent runs of each of these 12 specific model scenarios, while distributions of the empirically derived summary statistics were generated via bootstrapping. The goodness-of-fit tests were performed by summing the squared differences between 100 randomly sampled values from the empirical summary statistic distributions and from the distributions for each of the 12 specific model scenarios. This procedure was repeated 10,000 times to determine the frequency with which each specific model scenario was found to be best matching an empirical life-history pattern.

Subsequently, we assessed which selection pressure (spatial selection vs. local adaptation) best matched the observed latitudinal life-history patterns. For each of the three competing major model scenarios, we therefore summed the frequencies obtained in the previous step over all four trade-off balances. We thereby obtained three (one for each major model scenario) integrated frequencies, equally weighted over the four trade-off balances, for each of the five summary statistics. These integrated frequencies were then used to calculate Bayes's factors to infer-for each life-history pattern separately - the best-fitting major model scenario. This gave an idea of the most likely selection pres- sure shaping this life-history trait's variation along the latitudinal gradient. A Bayes's factor of 3 or more for a model comparison of model A versus B implies that model A is more strongly supported by the data (Kass and Raftery 1995). Thus, if, for example, model scenarios including range expansion gave a markedly better (Bayes's factor of three or more) fit than the stable range scenario for a specific life-history pattern, than spatial selection was inferred as the most likely selection pressure shaping the latitudinal pattern of this life-history trait.

\section{Results}

\section{Life-History Evolution along the Sampled Gradient}

Dispersal Propensity and Latency. Dispersal propensity and latency both significantly varied with latitude: dispersal propensity increased with latitude $\left(F_{1,2,235}=33.93, P<.001\right.$; fig. $2 A$ ), while dispersal latency showed the exact opposite trend $\left(F_{1,469}=12.16, P<.001\right.$; fig. $\left.2 B\right)$. Dispersal propensity and latency were density dependent, but this density dependence did not vary with latitude (propensity: $F_{2,2,230}=$ $0.03, P=.97$; latency: $F_{2,467}=2.71, P=.068$ ). There was no effect of host plant species on dispersal propensity $\left(F_{3,2,232}=1.85, P=.14\right)$ or dispersal latency $\left(F_{3,464}=\right.$ $0.60, P=.62)$.

Diapause Incidence. No correlation between latitude and diapause incidence was found $\left(F_{1,18.68}=0.05, P=.82\right.$; see sec. A4). Instead, diapause incidence was significantly af-

\section{A. aerial dispersal propensity}

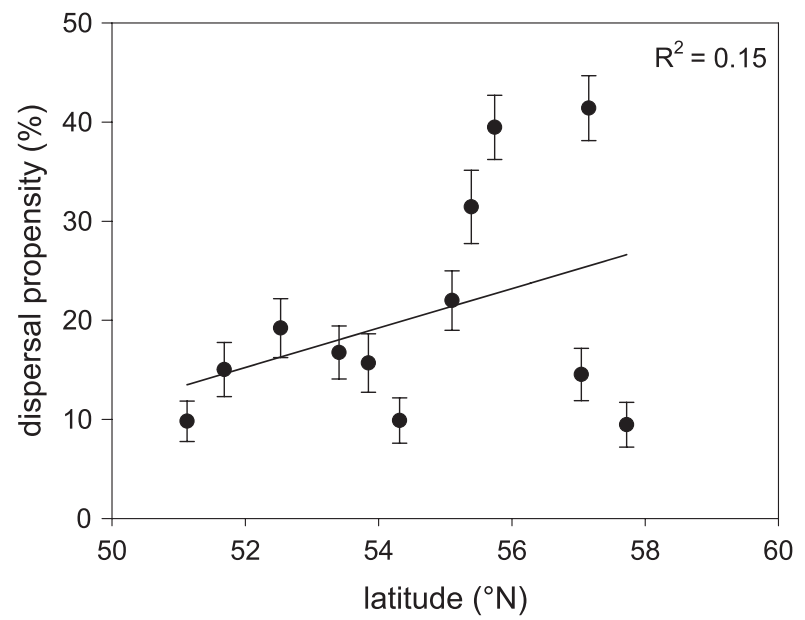

B. aerial dispersal latency

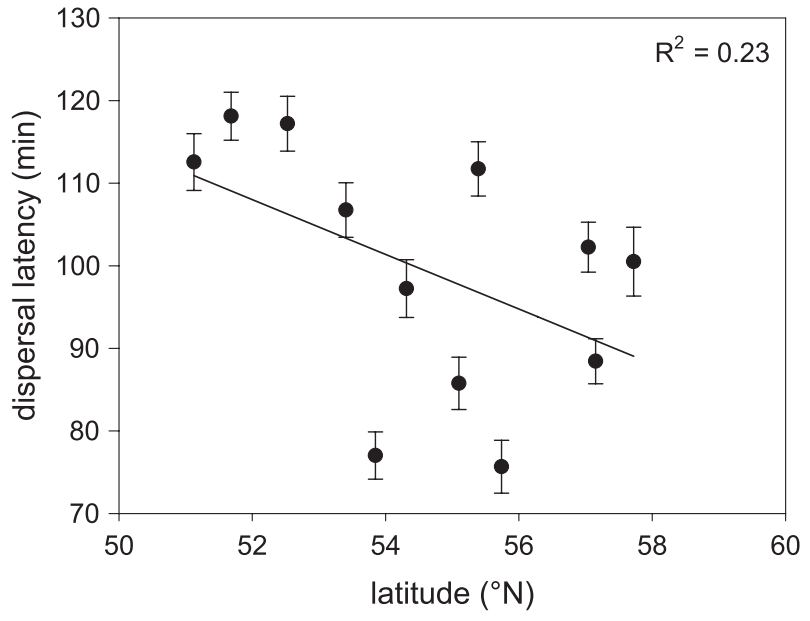

Figure 2: Dispersal propensity $(A)$ and dispersal latency $(B)$ for each sampled population along the latitudinal gradient. Populations means are given $\pm 1 \mathrm{SE}$ (bars). Regression lines (on population averages) are shown together with their $R^{2}$ values. 
fected by the host plant on which the mites were collected $\left(F_{3,50.13}=9.86, P<.001\right.$; see sec. A4 $)$.

Fecundity and Longevity. Lifetime fecundity $\left(F_{1,71}=14.75\right.$, $P<.001$; fig. $3 A)$ and longevity $\left(F_{1,65.1}=11.41, P=.0012\right.$; fig. $3 B$ ) both decreased significantly with latitude. For daily fecundity, however, no variation with latitude was found $\left(F_{1,68}=0.69, P=.41\right)$. Instead, daily fecundity was affected by the host plant species $\left(F_{3,69}=5.59, P=.0017\right.$; see sec. A4). For lifetime fecundity, no effects of host plant species were found $\left(F_{3,68}=1.62, P=.19\right)$. For longevity, there was a general effect of host plant species $\left(F_{3,66.3}=3.72\right.$, $P=.016$ ), but none of the adjusted $P$ values were significant in the pairwise post hoc Tukey tests.

Egg Survival, Juvenile Survival, and Development Time. The relative amount of hatched eggs increased significantly with latitude $\left(F_{1,103.1}=6.76, P=.011\right.$; fig. $\left.3 C\right)$, but the proportion of juvenile mites reaching the adult life stage showed no latitudinal pattern $\left(F_{1,1,315}=0.19, P=.67\right.$; see sec. A4). Furthermore, toward higher latitudes, female $\left(F_{1,66.1}=11.03, P=.0015\right)$ and male $\left(F_{1,62.1}=18.84, P<\right.$ $.001)$ spider mites had a significantly shorter development time (i.e., a faster development; fig. $3 D$ ). There was no effect of host plant species on the development time of females $\left(F_{3,57.4}=1.89, P=.14\right)$ or males $\left(F_{3,60}=2.21, P=.096\right)$ or on egg survival $\left(F_{3,44.38}=2.51, P=.071\right)$ or juvenile survival $\left(F_{3,1,312}=1.90, P=.13\right)$.

Fitting the expected sawtooth pattern (see sec. A4) instead of a linear predictor increased the amount of variance in development time explained from $36.4 \%$ to $80.9 \%$ in males $\left(F_{7,10}=6.06, P=.006\right)$ and from $13.3 \%$ to $61.2 \%$ in females $\left(F_{7,10}=2.26, P=.11\right)$. The deviance following segmented regression (sawtooth pattern) was (marginally) significantly lower than that for the linear pattern (onetailed $\chi^{2}$ tests; males: deviance $=2.70, P<.001$; females: deviance $=3.33, P=.054$ ).

Sex Ratio and Adult Size. Sex ratio (the proportion of males among offspring) increased significantly toward higher latitudes $\left(F_{1,61.97}=6.73, P=.012\right.$; fig. $\left.3 E\right)$. With increasing latitude, populations were thus increasingly male biased. Adult size, in contrast, did not vary with latitude $\left(F_{1,342}=1.19, P=.28\right.$; see sec. A4). Instead, the adult size of the female spider mites was significantly affected by the host plant species from which they were collected $\left(F_{3,343}=3.64, P=.013\right.$; see sec. A4). There was no effect of host plant species on sex ratio $\left(F_{3,50.9}=2.10, P=.11\right)$.

Intrinsic Growth Rate. Intrinsic growth rate decreased significantly toward higher latitudes $\left(F_{1,8}=6.20, P=.038\right.$; fig. $3 F)$.

\section{Inferring Mechanisms by Contrasting the Empirical Data with a Parameterized Individual-Based Model}

Three consistent (i.e., consistent over all the trade-off balances) patterns in life-history divergence along the latitudinal gradient emerged from the IBM: an increase in dispersal toward the range front in the range expansion scenarios, a stepwise decrease in voltinism toward the north in the scenarios with an environmental gradient, and an overall lower temperature for diapause termination than for diapause onset in all scenarios (see sec. A5). Our results furthermore show that the chosen tradeoff balance in our model (maximum effect on development vs. maximum effect on fecundity) affected voltinism and the relative investment in development versus fecundity (see sec. A2.6) and, as such, the goodness of fit of our three major model scenarios for the summary statistics in development time and intrinsic growth rate (table 1; fig. 4).

The goodness of fit for the five summary statistics clearly showed differences between the three competing major model scenarios (table 1; fig. 4). The stable range scenario poorly predicted the empirically observed dispersal propensity but provided some of the strongest supports for the pattern in development time. Overall, however, the stable range scenario performed rather badly. The scenario with range expansion in a homogeneous landscape showed a moderate overall fit but provided the strongest support for the empirical pattern in intrinsic growth rate. The scenario with range expansion along an environmental gradient resulted in the best overall fit, with the highest values for dispersal propensity and good to strong support for the patterns in intrinsic growth rate and development time. In terms of evolutionary scenario (range expansion vs. stable range and environmental gradient vs. no gradient), the range expansion scenarios clearly provided a much stronger support for the empirical pattern in dispersal than the stable range scenario (Bayes's factor $=40.15$ ), while no difference in support was found between the scenarios with and without an environmental gradient (Bayes's factor $=0.79$ ). In contrast, the best support for the empirical pattern in development time was provided by the scenarios with an environmental gradient (especially concerning the slope $[$ Bayes's factor $=1.99$ ] and amplitude [Bayes's factor $=$ 3.66] of the pattern), while no difference in support was found between model scenarios with or without range expansion (slope: Bayes's factor $=0.56$; amplitude: Bayes's factor $=0.56)$. Regarding intrinsic growth rate, no clear difference between the evolutionary scenarios was found (range expansion vs. stable range: Bayes's factor $=1.41$, gradient vs. no gradient: Bayes's factor $=0.74$ ), though the two best fits with the empirical data were provided by range expansion scenarios (see table 1). 
A. lifetime fecundity

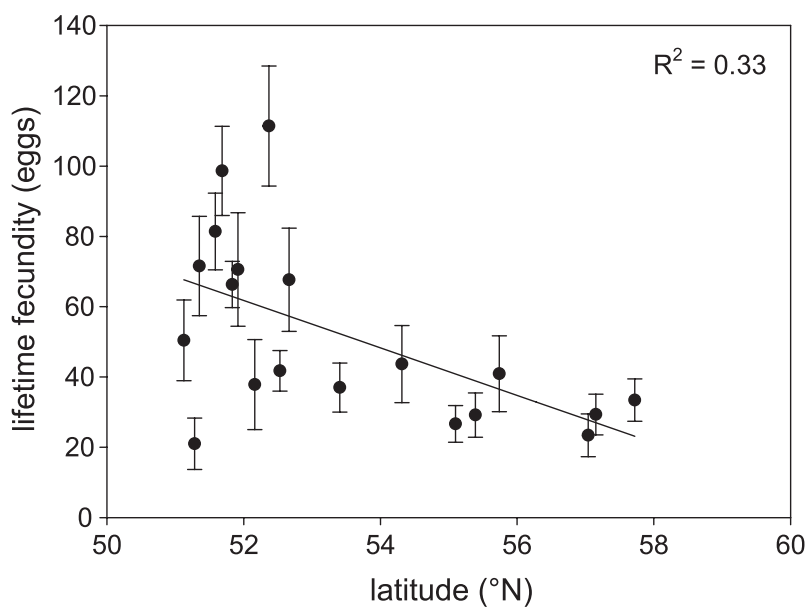

C. egg survival

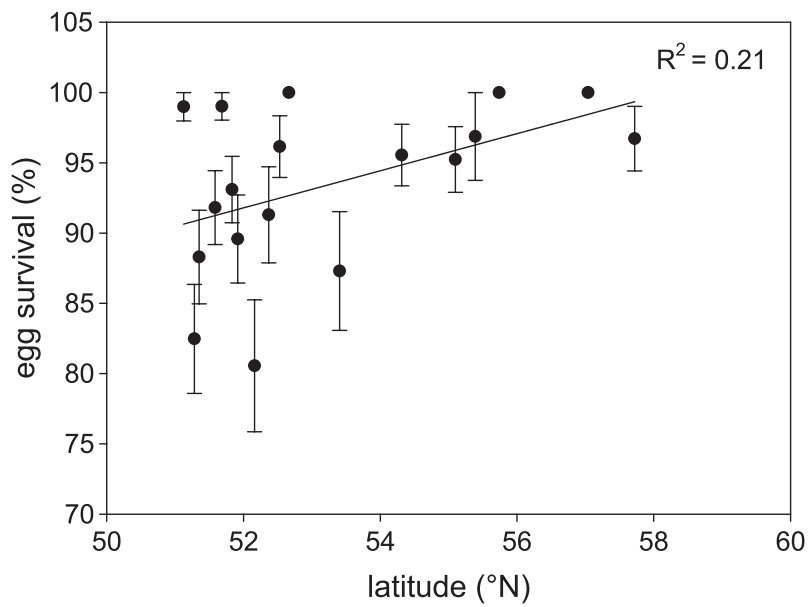

E. sex ratio

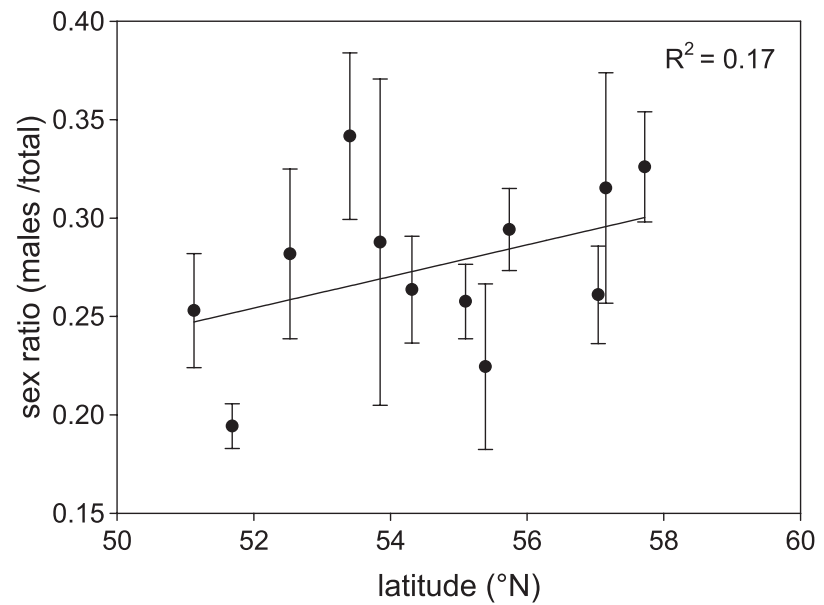

B. longevity

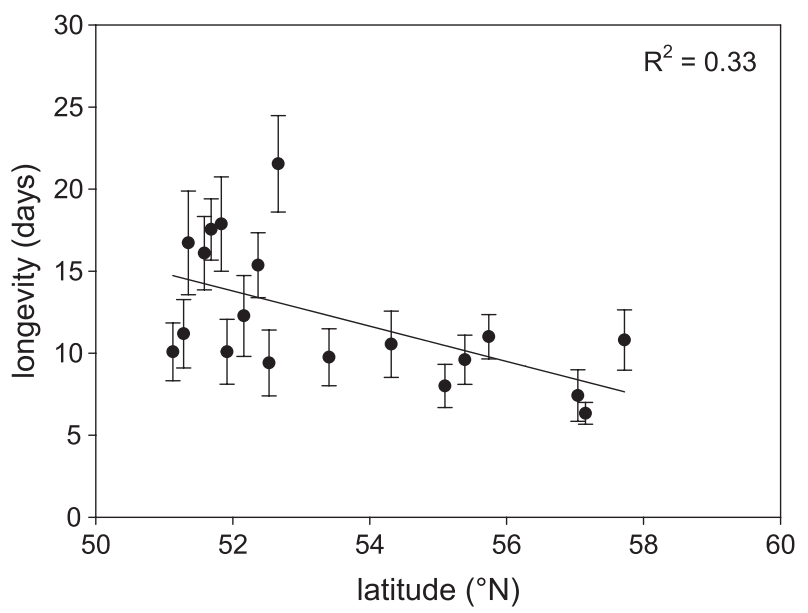

D. development time

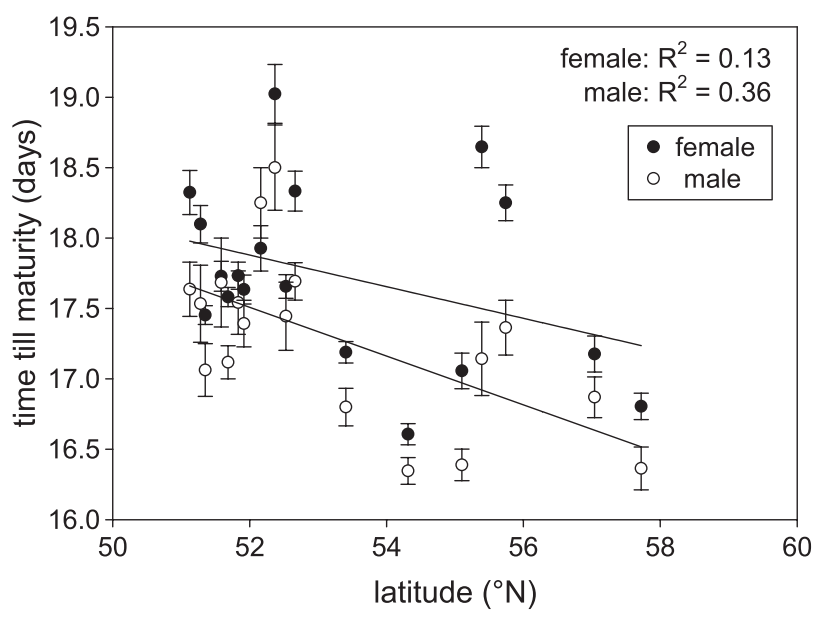

F. intrinsic growth rate

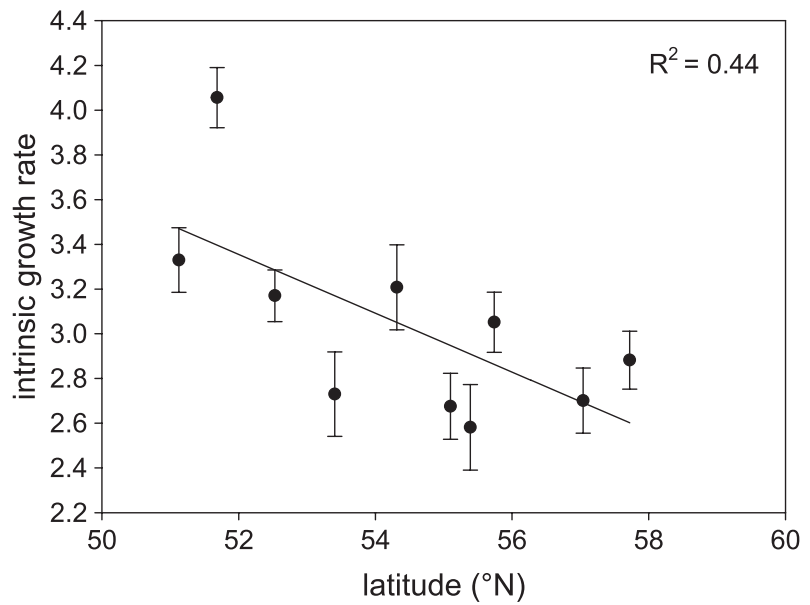

Figure 3: Lifetime fecundity $(A)$, longevity $(B)$, egg survival $(C)$, development time $(D)$, sex ratio $(E)$, and intrinsic growth rate $(F)$ for each sampled population along the latitudinal gradient. Population means are given \pm 1 SE (bars). Regression lines (based on population means) are shown together with their $R^{2}$ values. In $D$, development time is shown separately for females (filled circles) and males (open circles). 


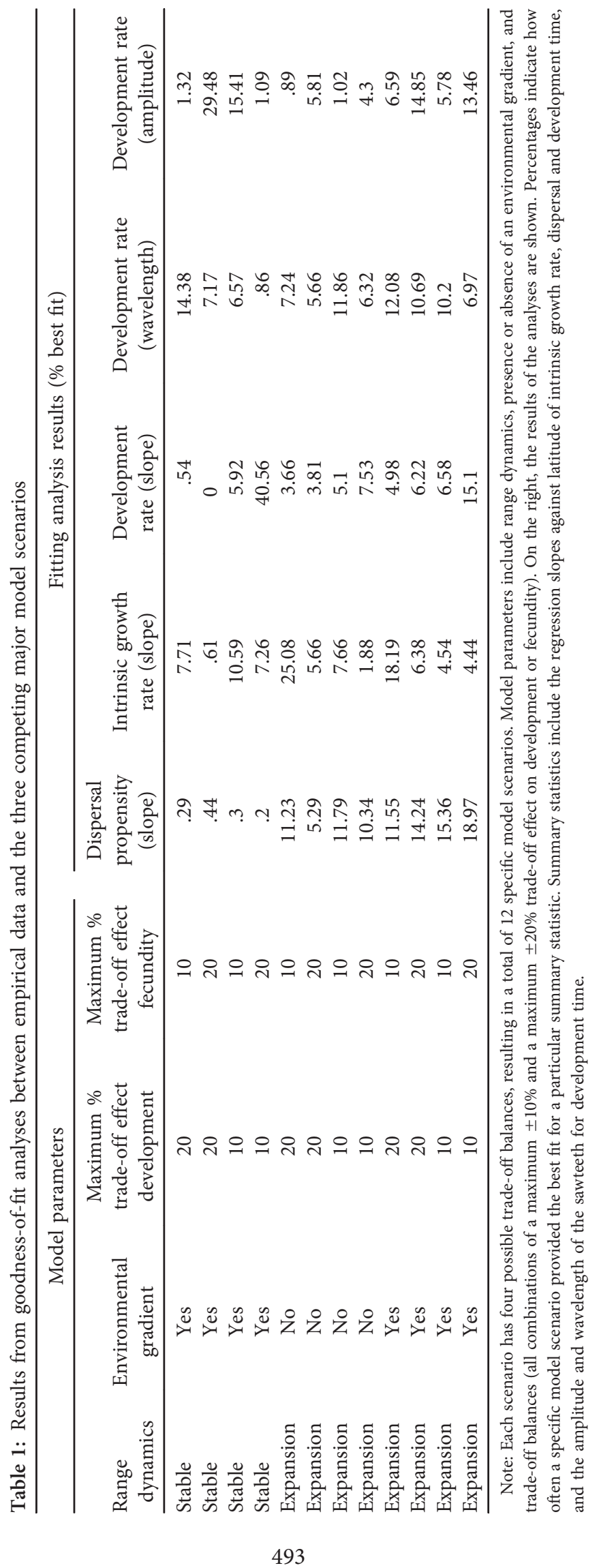




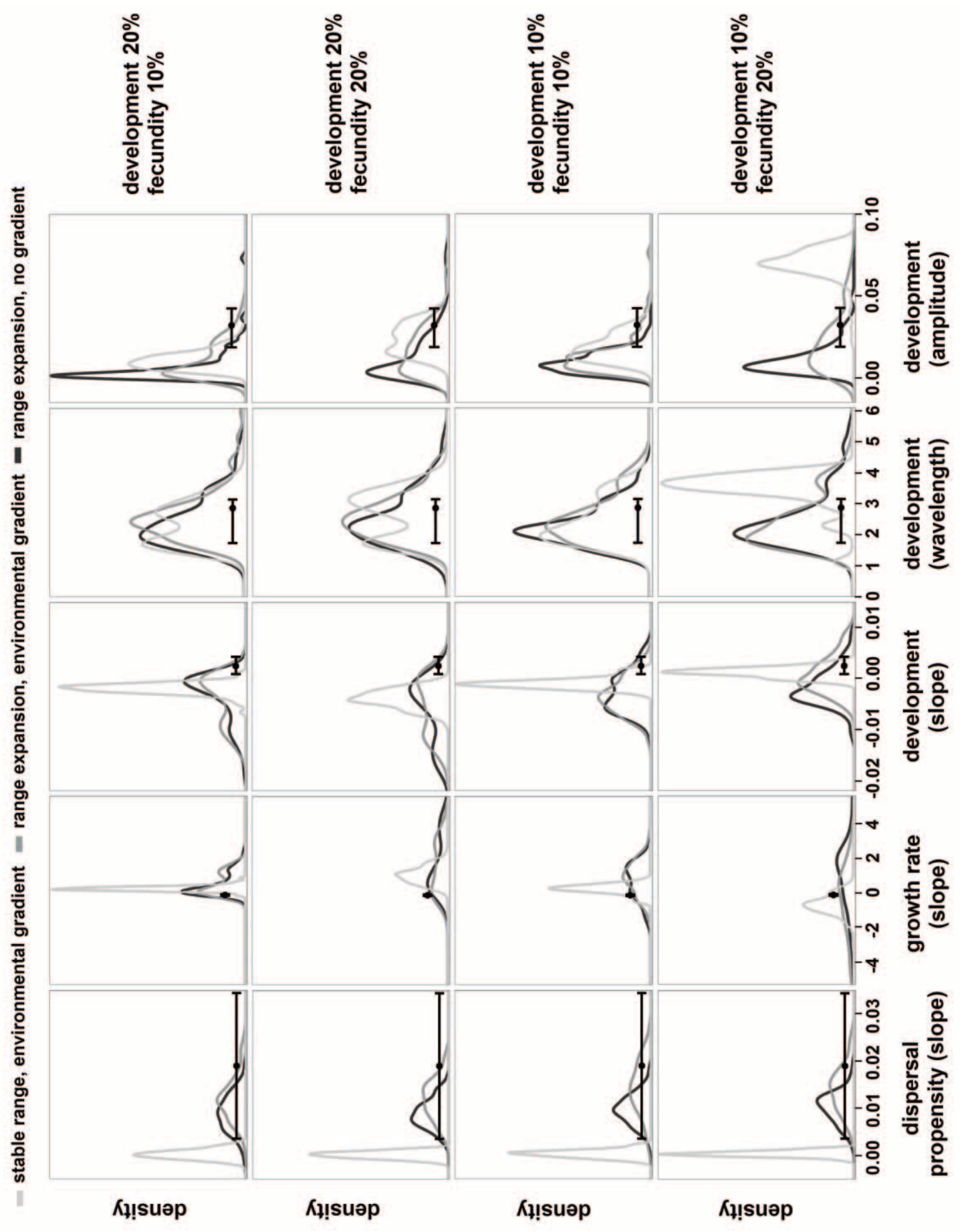

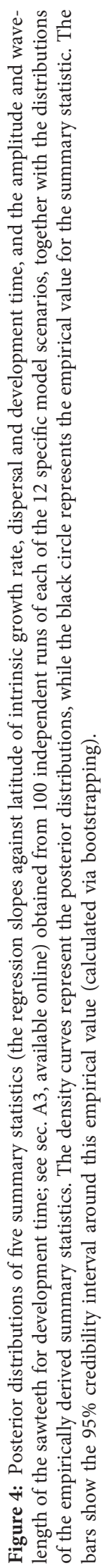




\section{Discussion}

Our common garden approach revealed considerable quantitative genetic divergence in life-history traits in populations of Tetranychus urticae that were sampled along a latitudinal gradient from range core to expansion front. Dispersal, sex ratio, egg survival, fecundity, longevity, development time, and the derived intrinsic growth rate showed strong latitudinal patterns. By means of pattern-oriented modeling, we demonstrated that local adaptation alone could not explain increased dispersal at high latitudes and thus that spatial selection likely must be in play. In contrast, latitudinal variation in development time was best explained by scenarios including local adaptation to the local climatic and seasonal conditions. For intrinsic growth rate, the trends were less clear, though the best fits were given by range expansion scenarios. Overall, local adaptation and spatial selection seem to have jointly shaped quantitative genetic divergence in the life history of this poleward-expanding arthropod.

The empirically observed increased dispersal at the range front is in line with several studies on postglacial range expansion (Cwynar and Macdonald 1987), invasions (Travis and Dytham 2002; Phillips et al. 2006; Huang et al. 2015), and climate change (Thomas et al. 2001; Travis et al. 2013). Because this pattern matched best with our range expansion scenarios, this indicates that dispersal ability is positively selected at the expansion front through the process of spatial selection and most likely not by adaptation to local environmental conditions (i.e., local temperature and growing season length in our model). Evolution of dispersal along a latitudinal gradient could, however, be equally affected by factors related to changes in habitat quality and connectivity (Bowler and Benton 2005), which were not included in our modeling framework. A decrease in habitat quality and connectivity, however, is theoretically expected to select against dispersal (Moran and Alexander 2014), so this would oppose our findings. It is interesting to notice that two populations at the outermost range limit at the peninsula of Denmark (which is surrounded by hostile matrix - the sea) are characterized by reduced dispersal (see fig. $2 A$ ). While these points do not obscure the general pattern, they potentially reflect an elastic range margin due to gradients of habitat availability and local extinction risk (Holt 2003; Kubisch et al. 2010; Henry et al. 2013). An additional factor leading to increased dispersal and colonization rates could be the ephemeral nature of range populations (Duputie and Massol 2013), as, for example, found in a plant species (Darling et al. 2008). However, in our study, host plants were readily available at the range front and expected to be exhausted more slowly than in the range core because of a lower intrinsic growth rate of the mites (see fig. $3 F$ ). Increased dispersal at the range edge could also have been caused by increased temporal variation in population sizes (McPeek and Holt 1992), resulting from harsh climatological conditions, especially during winter. In our model, however, this disturbance (see sec. A2.1) was implemented in both the stable and a range expansion scenario and can therefore not explain the difference in dispersal between these two scenarios. Spatial selection may thus be considered as a likely major driver of the evolution in dispersal in our study.

The empirically found latitudinal variation in development time (slope and sawtooth statistics) matched best with the scenarios that included adaptation toward an environmental gradient. The gradual shortening in the growing season from core to edge seems to have resulted in changes in voltinism and consequent abrupt changes in development time. Indeed, changes in development time are most effective for maintaining an optimal reproductive outcome when a restricted growing season leads to changes in voltinism (Roff 1980). The changes in development time did not cause changes in adult size in our study. This suggests that compensatory growth maintained a constant size at maturity, despite large changes in the length of the growing season (Conover et al. 2009). Interestingly, this might imply an increased foraging efficiency at the range margin and thus contradicts predictions of a dispersal-foraging trade-off, found during experimental evolution in a protist (Fronhofer and Altermatt 2015).

Concerning intrinsic growth rate, none of the three model scenarios gave a markedly better fit. The best fits, however, were provided by model scenarios with range expansion. Our empirically observed trend of a declining intrinsic growth rate with latitude, however, opposes theoretical expectations of evolution toward higher intrinsic growth rates at the expansion front, where on average lower population densities occur (Phillips 2009; Phillips et al. 2010). While Fronhofer and Altermatt (2015) showed that density is not always lower at the range margin, we suspect that it is in this case because of the overall shorter growing season and colder temperatures in the north. We therefore attribute the observed pattern in growth rate to trade-offs between fecundity and other life-history parameters. Indeed, the decline in intrinsic growth occurred despite faster development times and higher egg survival and was therefore most likely driven by strong reductions in fecundity. However, while some studies suggest that fecundity trades off with dispersal (e.g., Zera and Denno 1997; Hughes et al. 2003), others have failed to detect this or even found a positive correlation (e.g., Saastamoinen 2007; Therry et al. 2015). Furthermore, diapause incidence has been shown not to constrain changes in fecundity in T. urticae (Ito 2009). We conducted our study with a full life-history perspective and tested for population-level correlations between multiple life-history traits but did not detect any relevant trade-off (see sec. A6).

Because host plant variation covaried with the latitudinal gradient, our latitudinal patterns could have been confounded 
by patterns of local adaptation to the host plant species in the field. Tetranychus urticae is known to adapt to new host plant species within 10-15 generations (Magalhães et al. 2007), but we kept the mites in common garden for only two to five generations (except for the assessment of sex ratio), which is not sufficient to disrupt adaptation to a previous host plant species (Magalhães et al. 2011). However, we corrected for this potential bias in our analyses and found that host plant could have masked only a pure latitudinal effect for daily fecundity, where statistical models with host plant included did not show a latitudinal effect while models without did. In the case of diapause incidence, assessments were made almost immediately after mites were gathered in the field. Therefore, diapause incidence possibly still showed some environmentally induced phenotypic plasticity. Nevertheless, diapause is known to harbor a very strong genetic component (reviewed in Tauber et al. 1986).

By combining an empirical with a detailed, patternoriented modeling approach, this study is the first to demonstrate that local adaptation and evolution imposed by the process of range expansion can jointly shape quantitative genetic divergence during range expansion along a latitudinal gradient. We were able to show that local adaptation to the growing season probably affected development time, while the expansion process per se likely induced evolutionary divergence in dispersal and potentially also in intrinsic growth rate. In the current debate on the potential role of local adaptation versus phenotypic plasticity during range expansion, our results indicate that local adaptation has the potential to effectively drive rapid genotypic changes (Colautti and Barrett 2013). It can operate within the same ecological time frame as the process of spatial selection, together thrusting evolutionary change along the expansion front. To make reliable predictions for expanding populations, we should therefore acknowledge and take into account this interplay between both evolutionary forces.

\section{Acknowledgments}

This project was funded by the Fund for Scientific ResearchFlanders (FWO; project G.0610.11). D.B. and R.S. were supported by BelSpo Interuniversity Attraction Poles Project "Speedy." The computational resources (Stevin Supercomputer Infrastructure) and services used in this work were provided by the Flemish Supercomputer Center (VSC), funded by Ghent University, the Hercules Foundation, and the Flemish government (Department of Economy, Science, and Innovation). We are grateful to H. Matheve, R. Puls, J. Teunen, and J. Van den Berghe for their help during the sampling of the mites in the field and A. Alcantara, J. Hillaert, V. Vandomme, and A. Vanommeslaeghe for their short but useful assistance during the data gathering in the lab. We would also like to thank C. G. Eckert, E. Fronhofer, T. Hovestadt, E. Matthysen, M. Shawkey, D. Strubbe, J. Travis, and two anonymous reviewers for their useful comments on earlier versions of this manuscript.

\section{Literature Cited}

Agrawal, A. A., F. Vala, and M. W. Sabelis. 2002. Induction of preference and performance after acclimation to novel hosts in a phytophagous spider mite: adaptive plasticity? American Naturalist 159:553-565.

Baiser, B., H. L. Buckley, N. J. Gotelli, and A. M. Ellison. 2013. Predicting food-web structure with metacommunity models. Oikos 122:492-506.

Bancroft, J. S., and D. C. Margolies. 1999. An individual-based model of an acarine tritrophic system: lima bean, Phaseolus lunatus L., twospotted spider mite, Tetranychus urticae (Acari: Tetranychidae), and Phytoseiulus persimilis (Acari: Phytoseiidae). Ecological Modelling 123:161-181.

Blanckenhorn, W. U., and M. Demont. 2004. Bergmann and converse Bergmann latitudinal clines in arthropods: two ends of a continuum? Integrative and Comparative Biology 44:413-424.

Bowler, D. E., and T. G. Benton. 2005. Causes and consequences of animal dispersal strategies: relating individual behaviour to spatial dynamics. Biological Reviews 80:205-225.

Burton, O. J., B. L. Phillips, and J. M. J. Travis. 2010. Trade-offs and the evolution of life-histories during range expansion. Ecology Letters 13:1210-1220.

Carbonnelle, S., T. Hance, A. Migeon, P. Baret, S. Cros-Arteil, and M. Navajas. 2007. Microsatellite markers reveal spatial genetic structure of Tetranychus urticae (Acari: Tetranychidae) populations along a latitudinal gradient in Europe. Experimental and Applied Acarology 41:225-241.

Colautti, R. I., and S. C. H. Barrett. 2013. Rapid adaptation to climate facilitates range expansion of an invasive plant. Science 342:364366.

Conover, D. O., T. A. Duffy, and L. A. Hice. 2009. The covariance between genetic and environmental influences across ecological gradients: reassessing the evolutionary significance of countergradient and cogradient variation. Annals of the New York Academy of Sciences 1168:100-129.

Conover, D. O., and E. T. Schultz. 1995. Phenotypic similarity and the evolutionary significance of countergradient variation. Trends in Ecology and Evolution 10:248-252.

Crawley, M. J. 2007. Regression. Pages 387-448 in M. J. Crawley, ed. The R book. Wiley, Chichester.

Csillery, K., M. G. B. Blum, O. E. Gaggiotti, and O. Francois. 2010. Approximate Bayesian computation (ABC) in practice. Trends in Ecology and Evolution 25:410-418.

Cwynar, L. C., and G. M. Macdonald. 1987. Geographical variation of lodgepole pine in relation to population history. American Naturalist 129:463-469.

Darling, E., K. E. Samis, and C. G. Eckert. 2008. Increased seed dispersal potential towards geographic range limits in a Pacific coast dune plant. New Phytologist 178:424-435.

Denno, R. F., and M. A. Peterson. 1995. Density-dependent dispersal and its consequences for population dynamics. Pages 113-130 in N. Cappuccino and P. W. Price, eds. Population dynamics: new approaches and synthesis. Academic Press, London. 
De Roissart, A., S. Wang, and D. Bonte. 2015. Spatial and spatiotemporal variation in metapopulation structure affects population dynamics in a passively dispersing arthropod. Journal of Animal Ecology 84:1565-1574.

Duputie, A., and F. Massol. 2013. An empiricist's guide to theoretical predictions on the evolution of dispersal. Interface Focus 3:20130028.

Fronhofer, E. A., and F. Altermatt. 2015. Eco-evolutionary feedbacks during experimental range expansions. Nature Communications 6:6844.

Gotoh, T., A. Suwa, Y. Kitashima, and H. A. Rezk. 2004. Developmental and reproductive performance of Tetranychus pueraricola Ehara and Gotoh (Acari: Tetranychidae) at four constant temperatures. Applied Entomology and Zoology 39:675-682.

Grimm, V., E. Revilla, U. Berger, F. Jeltsch, W. M. Mooij, S. F. Railsback, H. H. Thulke, et al. 2005. Pattern-oriented modeling of agent-based complex systems: lessons from ecology. Science 310:987-991.

Hargreaves, A. L., S. F. Bailey, and R. A. Laird. 2015. Fitness declines towards range limits and local adaptation to climate affect dispersal evolution during climate-induced range shifts. Journal of Evolutionary Biology 28:1489-1501.

Hargreaves, A. L., and C. G. Eckert. 2014. Evolution of dispersal and mating systems along geographic gradients: implications for shifting ranges. Functional Ecology 28:5-21.

Hargreaves, A. L., K. E. Samis, and C. G. Eckert. 2014. Are species' range limits simply niche limits writ large? a review of transplant experiments beyond the range. American Naturalist 183:157-173.

Harrison, R. G. 1980. Dispersal polymorphisms in insects. Annual Review of Ecology and Systematics 11:95-118.

Henry, R. C., G. Bocedi, and J. M. J. Travis. 2013. Eco-evolutionary dynamics of range shifts: elastic margins and critical thresholds. Journal of Theoretical Biology 321:1-7.

Hill, J. K., H. M. Griffiths, and C. D. Thomas. 2011. Climate change and evolutionary adaptations at species' range margins. Annual Review of Entomology 56:143-159.

Holt, R. D. 2003. On the evolutionary ecology of species' ranges. Evolutionary Ecology Research 5:159-178.

Huang, F., S. Peng, B. Chen, H. Liao, Q. Huang, Z. Lin, and G. Liu. 2015. Rapid evolution of dispersal-related traits during range expansion of an invasive vine Mikania micrantha. Oikos 124:1023-1030.

Hughes, C. L., J. K. Hill, and C. Dytham. 2003. Evolutionary tradeoffs between reproduction and dispersal in populations at expanding range boundaries. Proceedings of the Royal Society B 270:S147S150.

Ito, K. 2009. Does artificial selection on diapause incidence cause correlational changes in other life-history traits? case study in a spider mite population. Entomologia Experimentalis et Applicata 130:266-274.

Kass, R. E., and A. E. Raftery. 1995. Bayes factors. Journal of the American Statistical Association 90:773-795.

Kivela, S. M., P. Valimaki, D. Carrasco, M. I. Maenpaa, and J. Oksanen. 2011. Latitudinal insect body size clines revisited: a critical evaluation of the saw-tooth model. Journal of Animal Ecology 80:1184-1195.

Krainacker, D. A., and J. R. Carey. 1989. Reproductive limits and heterogeneity of male twospotted spider mites. Entomologia Experimentalis et Applicata 50:209-214.

Kubisch, A., R. D. Holt, H. J. Poethke, and E. A. Fronhofer. 2014. Where am I and why? synthesizing range biology and the ecoevolutionary dynamics of dispersal. Oikos 123:5-22.
Kubisch, A., T. Hovestadt, and H.-J. Poethke. 2010. On the elasticity of range limits during periods of expansion. Ecology 91:3094-3099.

Lehmann, P., A. Lyytinen, S. Piiroinen, and L. Lindstrom. 2014. Northward range expansion requires synchronization of both overwintering behaviour and physiology with photoperiod in the invasive Colorado potato beetle (Leptinotarsa decemlineata). Oecologia (Berlin) 176:57-68.

- 2015. Latitudinal differences in diapause related photoperiodic responses of European Colorado potato beetles (Leptinotarsa decemlineata). Evolutionary Ecology 29:269-282.

Levy, R. C., G. M. Kozak, C. B. Wadsworth, B. S. Coates, and E. B. Dopman. 2015. Explaining the sawtooth: latitudinal periodicity in a circadian gene correlates with shifts in generation number. Journal of Evolutionary Biology 28:40-53.

Li, J. B., and D. C. Margolies. 1994. Responses to direct and indirect selection on aerial dispersal behavior in Tetranychus urticae. Heredity $72: 10-22$.

Magalhães, S., E. Blanchet, M. Egas, and I. Olivieri. 2011. Environmental effects on the detection of adaptation. Journal of Evolutionary Biology 24:2653-2662.

Magalhães, S., J. Fayard, A. Janssen, D. Carbonell, and I. Olivieri. 2007. Adaptation in a spider mite population after long-term evolution on a single host plant. Journal of Evolutionary Biology 20: 2016-2027.

McPeek, M. A., and R. D. Holt. 1992. The evolution of dispersal in spatially and temporally varying environments. American Naturalist 140:1010-1027.

Mitikka, V., and I. Hanski. 2010. Pgi genotype influences flight metabolism at the expanding range margin of the European map butterfly. Annales Zoologici Fennici 47:1-14.

Moran, E. V., and J. M. Alexander. 2014. Evolutionary responses to global change: lessons from invasive species. Ecology Letters 17: 637-649.

Muggeo, V. M. R. 2003. Estimating regression models with unknown break-points. Statistics in Medicine 22:3055-3071.

2008. segmented: an R package to fit regression models with broken-line relationships. R News 8:20-25.

North, A., S. Cornell, and O. Ovaskainen. 2011. Evolutionary responses of dispersal distance to landscape structure and habitat loss. Evolution 65:1739-1751.

Parmesan, C. 2006. Ecological and evolutionary responses to recent climate change. Annual Review of Ecology, Evolution, and Systematics 37:637-669.

Perkins, A. T., B. L. Phillips, M. L. Baskett, and A. Hastings. 2013. Evolution of dispersal and life history interact to drive accelerating spread of an invasive species. Ecology Letters 16:1079-1087.

Phillips, B. L. 2009. The evolution of growth rates on an expanding range edge. Biology Letters 5:802-804.

Phillips, B. L., G. P. Brown, and R. Shine. 2010. Life-history evolution in range-shifting populations. Ecology 91:1617-1627.

Phillips, B. L., G. P. Brown, J. K. Webb, and R. Shine. 2006. Invasion and the evolution of speed in toads. Nature 439:803.

Piiroinen, S., T. Ketola, A. Lyytinen, and L. Lindstrom. 2011. Energy use, diapause behaviour and northern range expansion potential in the invasive Colorado potato beetle. Functional Ecology 25:527536.

Richardson, D. M., and M. Rejmanek. 2011. Trees and shrubs as invasive alien species: a global review. Diversity and Distributions 17: 788-809.

Roff, D. 1980. Optimizing development time in a seasonal environment: the ups and downs of clinal variation. Oecologia (Berlin) 45:202-208. 
RStudio Team. 2015. RStudio: integrated development for R. RStudio, Boston. http://www.rstudio.com/.

Saastamoinen, M. 2007. Mobility and lifetime fecundity in new versus old populations of the Glanville fritillary butterfly. Oecologia (Berlin) 153:569-578.

Sabelis, M. W. 1981. Biological control of two-spotted spider mites using phytoseiid predators. I. Modelling the predator-prey interaction at the individual level. Centre for Agricultural Publishing and Documentation, Wageningen.

SAS Institute. 2013. SAS software. Version 9.4. SAS Institute, Cary, NC

Shine, R., G. P. Brown, and B. L. Phillips. 2011. An evolutionary process that assembles phenotypes through space rather than through time. Proceedings of the National Academy of Sciences of the USA 108:5708-5711.

Tauber, M. J., C. A. Tauber, and S. Masaki. 1986. Seasonal adaptations of insects. Oxford University Press, Oxford.

Therry, L., D. Bonte, and R. Stoks. 2015. Higher investment in flight morphology does not trade off with fecundity estimates in a poleward range-expanding damselfly. Ecological Entomology 40:133-142.

Therry, L., E. Lefevre, D. Bonte, and R. Stoks. 2014a. Increased activity and growth rate in the non-dispersive aquatic larval stage of a damselfly at an expanding range edge. Freshwater Biology 59:1266-1277.

Therry, L., V. Nilsson-Ortman, D. Bonte, and R. Stoks. 2014b. Rapid evolution of larval life history, adult immune function and flight muscles in a poleward-moving damselfly. Journal of Evolutionary Biology 27:141-152.

Therry, L., A. Zawal, D. Bonte, and R. Stoks. 2014c. What factors shape female phenotypes of a poleward-moving damselfly at the edge of its range? Biological Journal of the Linnean Society 112:556-568.

Thomas, C. D., E. J. Bodsworth, R. J. Wilson, A. D. Simmons, Z. G. Davies, M. Musche, and L. Conradt. 2001. Ecological and evolutionary processes at expanding range margins. Nature 411:577-581.

Travis, J. M. J., M. Delgado, G. Bocedi, M. Baguette, K. Barton, D. Bonte, I. Boulangeat, et al. 2013. Dispersal and species' responses to climate change. Oikos 122:1532-1540.

Travis, J. M. J., and C. Dytham. 2002. Dispersal evolution during invasions. Evolutionary Ecology Research 4:1119-1129.

Van Petegem, K. H. P., J. Boeye, R. Stoks, and D. Bonte. 2016. Data from: Spatial selection and local adaptation jointly shape life-history evolution during range expansion. American Naturalist, Dryad Digital Repository, http://dx.doi.org/10.5061/dryad.n0c67.

Van Petegem, K. H. P., J. Pétillon, D. Renault, N. Wybouw, T. Van Leeuwen, and D. Bonte. 2015. Empirically simulated spatial sorting points at fast epigenetic changes in dispersal behaviour. Evolutionary Ecology 29:299-310.

Wiegand, T., and K. A. Moloney. 2014. Handbook of spatial pointpattern analysis in ecology. CRC, Boca Raton, FL.

Young, S. S. Y., D. L. Wrensch, and M. Kongchuensin. 1986. Control of sex-ratio by female spider-mites. Entomologia Experimentalis et Applicata 40:53-60.

Zera, A. J., and R. F. Denno. 1997. Physiology and ecology of dispersal polymorphism in insects. Annual Review of Entomology 42:207-230.

\section{References Cited Only in the Online Appendixes}

Bitume, E. V., D. Bonte, S. Magalhaes, G. S. Martin, S. Van Dongen, F. Bach, J. M. Anderson, et al. 2011. Heritability and artificial se- lection on ambulatory dispersal distance in Tetranychus urticae: effects of density and maternal effects. PLOS ONE 6:e26927.

Bitume, E. V., D. Bonte, O. Ronce, I. Olivieri, and C. M. Nieberding. 2014. Dispersal distance is influenced by parental and grandparental density. Proceedings of the Royal Society B 281:20141061.

Breslow, N. E. and D. G. Clayton. 1993. Approximate inference in generalized linear mixed models. Journal of the American Statistical Association 88:9-25.

Fronhofer, E. A., J. M. Stelz, E. Lutz, H. J. Poethke, and D. Bonte. 2014. Spatially correlated extinctions select for less emigration but larger dispersal distances in the spider mite Tetranychus urticae. Evolution 68:1838-1844.

Griffing, B. 1982. A theory of natural selection incorporating interaction among individuals. 10. Use of groups consisting of a mating pair together with haploid and diploid caste members. Journal of Theoretical Biology 95:199-223.

Hartl, D. L. 1971. Some aspects of natural selection in arrhenotokous populations. American Zoologist 11:309-325.

Havron, A., D. Rosen, Y. Rossler, and J. Hillel. 1987. Selection on the male hemizygotic genotype in arrhenotokous insects and mites. Entomophaga 32:261-268.

Lactin, D. J., N. J. Holliday, D. L. Johnson, and R. Craigen. 1995. Improved rate model of temperature-dependent development by arthropods. Environmental Entomology 24:68-75.

Lewis, Z., P. M. Brakefield, and N. Wedell. 2010. Speed or sperm: a potential trade-off between development and reproduction in the butterfly, Bicyclus anynana (Lepidoptera: Nymphalidae). European Journal of Entomology 107:55-59.

Logan, J. A., D. J. Wollkind, S. C. Hoyt, and L. K. Tanigoshi. 1976. Analytic model for description of temperature-dependent rate phenomena in arthropods. Environmental Entomology 5:1133-1140.

Nunney, L. 1996. The response to selection for fast larval development in Drosophila melanogaster and its effect on adult weight: an example of a fitness trade-off. Evolution 50:1193-1204.

SAS Institute. 2008. SAS/STAT 9.2 user's guide. SAS Institute, Cary, NC.

Schneider, C. A., W. S. Rasband, and K. W. Eliceiri. 2012. NIH Image to ImageJ: 25 years of image analysis. Nature Methods 9:671675.

Tien, N. S. H., M. W. Sabelis, and M. Egas. 2011. Ambulatory dispersal in Tetranychus urticae: an artificial selection experiment on propensity to disperse yields no response. Experimental and Applied Acarology 53:349-360.

Tsikliras, A. C., E. Antonopoulou, and K. I. Stergiou. 2007. A phenotypic trade-off between previous growth and present fecundity in round sardinella Sardinella aurita. Population Ecology 49:221-227.

Verbeke, G., and G. Molenberghs. 2000. Linear mixed models for longitudinal data. Springer, New York.

Wolfinger, R. D., and M. A. O'Connell. 1993. Generalized linear mixed models: a pseudo-likelihood approach. Journal of Statistical Computation and Simulation 48:233-243.

Yadav, P., and V. K. Sharma. 2014. Correlated changes in life history traits in response to selection for faster pre-adult development in the fruit fly Drosophila melanogaster. Journal of Experimental Biology 217:580-589. 